\title{
ANTECEDENTES DA INTENÇÃO DE RECOMPRA EM APLICATIVO DE HOSPEDAGEM
}

ANTECEDENTES OF REPURCHASE INTENTION IN HOST APP

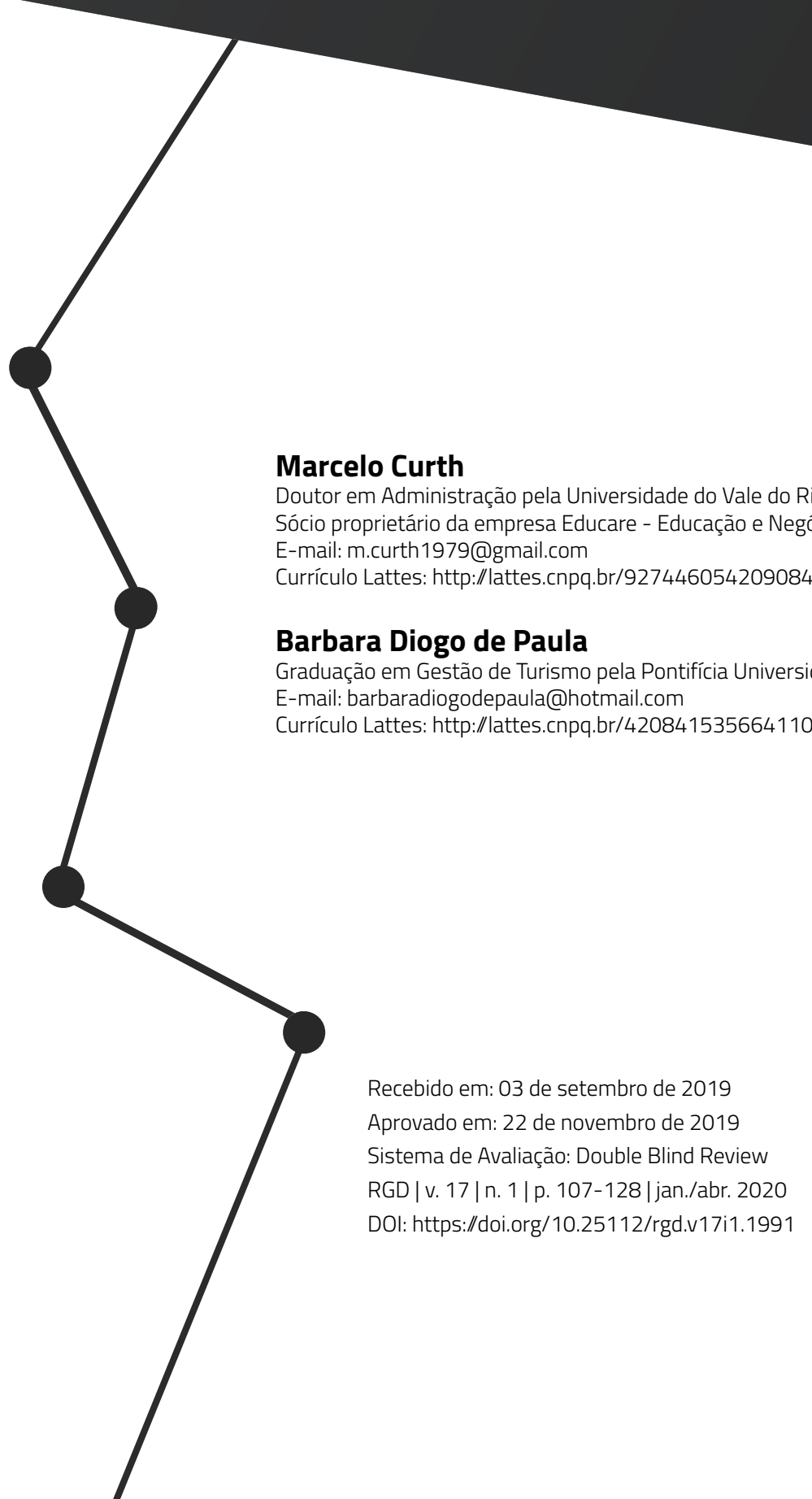




\section{RESUMO}

O avanço tecnológico tem possibilitado o desenvolvimento de novos formatos de negócios no contexto virtual, sendo os casos dos aplicativos de reserva de locais e de experiências. No entanto, ainda são criadas barreiras no consumo em tais aplicativos, por conta da confiança dos participantes. Nesse sentido, o artigo tem como objetivo identificar a influência da utilidade percebida, da facilidade de uso e da confiança na empresa e em terceiros na intenção de recompra em aplicativos de hospedagem. Para isso, foi realizada uma survey com 176 usuários de um aplicativo de hospedagem (Airbnb) e a análise realizada por meio da modelagem de equações estruturais (MEE). Os resultados apresentaram que a utilidade percebida e facilidade de uso influenciam positivamente na intenção de recompra, porém a confiança na empresa e em terceiro não foi positiva. Além disso, a utilidade percebida influenciou positivamente a confiança na empresa e em terceiros, o que não ocorreu com a facilidade de uso. A pesquisa contribuiu para uma reflexão a respeito da nova economia ou da economia compartilhada, possibilitando reflexões para as empresas no que tange à importância da percepção de utilidade de seus aplicativos.

Palavras-chave: Percepção de utilidade. Facilidade de uso. Confiança. Intenção de recompra. Aplicativos móveis.

\section{ABSTRACT}

The technological advance has made possible the development of new business formats in the virtual context, being the cases of applications of places reservation and experiences. However, consumption barriers are still created in such applications, because of the confidence of the participants. In this sense, the article aims to identify the influence of perceived utility, ease of use and trust in the company and third parties in the intention of repurchase in hosting applications. For this, a survey was carried out with 176 users of a hosting application (Airbnb) and the analysis performed through structural equation modeling (SEM). The results showed that the perceived utility and ease of use positively influence the repurchase intention, but confidence in the company and in the third one was not positive. In addition, perceived utility positively influenced trust in the company and in others, which did not occur with ease of use. The research contributed to a reflection about the new economy or the shared economy, allowing reflections for companies on the importance of the perception of utility of their applications.

Keywords: Perceived usefulness. Perceived ease of use. Trust. Repurchase intention. Mobile apps. 


\section{INTRODUÇÃO}

A tecnologia da comunicação móvel sofreu grandes avanços nos últimos anos, com celulares cada vez mais evoluídos, possibilitando os usuários a conectar-se na internet por um baixo custo, facilitando o acesso a comunicação e diversos serviços (CURTH, 2018). Partindo desse cenário, surgiram novas tecnologias, como os aplicativos móveis ou app que hoje vem ganhando grande espaço tanto para as empresas quanto para os consumidores (TURBAN; KING, 2004; CURTH, 2018).

Por conta do crescimento da utilização dos dispositivos móveis e do referido aumento do número de participações dos usuários via apps, surgem estudos que buscam melhor entendimento acerca do comportamento de consumo no universo da tecnologia mobile (CURTH, 2018).

O consumidor busca o ambiente on-line para economizar tempo, ter maior facilidade para encontrar o produto/serviço, ter a sensação de prazer ao comprar, maior facilidade de comparar preços e a utilização dos serviços pós-venda (SCHAUPP; BÉLANGER, 2005). Além disso, a experiência de compra compreende confiança, prazo de entrega, percepções e sentimentos durante e após a compra. No entanto, percebe-se a lacuna para a inclusão de elementos de confiança nas transações realizadas no ambiente virtual/mobile, considerando a segurança das informações, a empresa provedora dos serviços e terceiros (MCCOLE; RAMSEY; WILLIAMS, 2010; WU; CHEN; CHUNG, 2010; ROHDEN; MATOS; CURTH, 2016; GARCIA; CELSO; CURTH, 2017) com teorias que abordem a utilização de construtos já utilizados de intenção de utilização de tecnologia (DAVIS, 1989; DAVIS et al. 1989; HU et al. 1989; VENKATESH, 1999; VENKATESH; DAVIS, 2000; VENKATESH et al. 2003).

Dessa forma, chega-se ao problema da pesquisa que trata de entender qual o impacto das percepções de utilidade, facilidade de uso e confiança dos envolvidos na intenção de realizar novas transações em aplicativos de viagem e experiência?

Os objetivos gerais dessa pesquisa são: Identificar a influência da utilidade e facilidade de uso na intenção de recompra bem como a influência da confiança na empresa e em terceiros na intenção de recompra dos usuários. Já os objetivos específicos tratam da a) identificar a intenção de recompra dos usuários b) identificar a confiança na empresa e em terceiros e c) identificar o perfil dos respondentes.

Para melhor ilustrar a pesquisa, a empresa escolhida para ser a referência do estudo é a empresa de hospedagem Airbnb, pois trata-se de um exemplo de empresa que oferece serviço de hospedagem em diversas cidades do mundo. A empresa foi fundada em novembro de 2008 em São Francisco na Califórnia. o Airbnb é especializado em economia compartilhada, permitindo que o indivíduo possa alugar sua casa ou apartamento pelo site ou aplicativo dando a oportunidade de o turista reservar um alojamento em 192 países e assim incentivando o encontro de pessoas em diversos lugares do mundo. 
Deste modo, o aplicativo de hospedagem Airbnb é um exemplo de empresa que explora esse mercado de hospedagem utilizando a internet e o uso de aplicativo como canal de venda, sendo a forma mais pura de comércio eletrônico, uma vez que o processo de venda é realizado on-line. $\mathrm{O}$ aplicativo do Airbnb é um dos mais usados para reservas de alojamento, pois por meio dele é possível filtrar por preço, localização, data e até ver opiniões de outras pessoas que tenham se hospedado no local pesquisado.

A pesquisaéimportante, pois por meio dela podemos identificar como a confiança pode influenciar no comportamento dos consumidores de aplicativos de viagem e entender as facilidades que os avanços tecnológicos trouxeram para quem planejauma viagem, além das relações com influentes da intenção de recompra no ambiente virtual.

\section{REFERENCIAL TEÓRICO}

\subsection{TECNOLOGIA MOBILE}

É inegável que a tecnologia digital esteja cada vez mais presente no cotidiano da sociedade e gerando cada vez mais facilidades e oportunidades para a população, gerando mudanças na forma de trabalho e novos hábitos de consumo. As compras on-line estão ganhando força no mercado digital, muitas empresas virtuais surgindo e outras se reinventando para se adaptar a esse novo mercado tecnológico.

Os celulares estão cada vez mais avançados e as empresas se adaptando devido as novas oportunidades de mercado, lançando aplicativos de celular onde a população consegue efetuar suas compras pelo próprio dispositivo móvel, seja pela web ou aplicativo de celular.

Segundo dados do Ministério do Turismo, dos 127 milhões de usuários do Facebook, 122 milhões usam a plataforma pelo celular. Desses, cerca de $22 \%$ navegam pelo celular para concluir viagens (BRASIL, 2018). O aplicativo é um programa desenvolvido para ser instalado em um dispositivo eletrônico móvel, ele pode ser gratuito ou pago. O mercado de aplicativo móvel é muito promissor e vem crescendo rapidamente.

Muitas empresas estão investindo seu negócio em vendas on-line por meio de aplicativos, pois os apps estão tomando conta do mercado, devido a praticidade de comprar sem sair de casa e por estar tão próximo do consumido, como o celular por exemplo que pode ser usado a qualquer momento do dia para a compra por meio de aplicativos. Nesse contexto, Martha (2010) afirma que o futuro é mobile porque a natureza humana é mobile e as tecnologias digitais não apenas estão eliminando todas as restrições que nos impedem de exercitar a nossa mobilidade instintiva, mas também estão alavancando-a. 
A era mobile está crescendo tão rápido e a população acaba virando refém e ficando conectada 24 horas por dia, seja pelas redes sócias, trabalhos ou em compras on-line. Vassos (1997) afirmou muitos antes da tecnologia ter tamanho espaço na sociedade: A internet tem o potencial de trazer profundas mudanças para a sociedade. Os efeitos serão profundos porque a tecnologia tem tal capacidade. A internet terá impacto sobre muitos setores porque é capaz de transmitir texto, gráficos, conversas telefônicas, sinais de áudio, música e vídeos.

Estamos conectados diariamente, fazendo compras, trabalhando e usando redes sociais no aparelho celular. Sobre os avanços tecnológicos os autores Turban e King (2004) ressaltam que o m-commerce se baseia no fato de que os usuários carregam um telefone celular ou outro dispositivo móvel para onde quer que vão. Mobilidade implica portabilidade. Por conseguinte, o usuário pode iniciar um contato em tempo real com determinado sistema onde quer que esteja.

Diante desse cenário, as empresas passaram a ver a oportunidade de investir em comercio eletrônico e principalmente no mercado de aplicativos para compras que se mostra cada vez mais promissor e vantajoso para ambas as partes. Torres (2011) destaca que o aplicativo é o componente responsável pela maioria das inovações e usos diferenciados considerando aspectos como usabilidade e interatividade, tornando o dispositivo móvel mais flexível e útil.

Portanto, o mercado de aplicativos está cada vez mais ganhando espaço na sociedade, trazendo suas facilidades, agilidades e dando a oportunidade de o consumidor buscar o serviço/produto mais de acordo com o pretendido evitando de precisar se deslocar até uma loja fixa.

\subsection{TECNOLOGIA EM MEIOS DE HOSPEDAGEM}

Com os avanços tecnológicos muitas oportunidades surgiram na exploração do turismo e de cidades por meio de hospedagens (Ruiz, et al. 2019), tanto para quem trabalha quanto quem busca usufruir do serviço. Diante desse cenário promissor, a Airbnb vem ganhando espaço e investindo no mercado virtual, todas as vendas são feitas on-line e o consumidor pode buscar um alojamento de acordo com sua necessidade, seja ela localização, preço ou qualidade em qualquer lugar do mundo.

Segundo Molina (2003), a concepção de empresas virtuais não trata só das mesmas empresas, com um significativo agregado tecnológico. Trata-se de uma nova categoria de empresa, com uma nova estrutura, funções e produtos/serviços no âmbito de mercados que também experimentaram transformações nesses níveis.

As tendências para o mercado de hospedagem ressaltam os viajantes procurando praticidade e baixo custo. De acordo com Bahl (2003), os viajantes do futuro serão receptivos para novas tecnologias e serviços que facilitem a viagem e reduzam os custos. 
Segundo Buhalis (1994), a atividade turística tem recebido um apoio principal, que são as Tecnologias da Informação. É perceptível a necessidade de transmissão de informação existente por parte do turismo. Então, de acordo com a evolução tecnológica, a atividade turística se fundiu com as Tecnologias de Informação (TI).

Turban e King (2004) abordam sobre a mobilidade que impulsionou o mercado de celulares cada vez mais inteligentes e com Não importa se você precisa de um apartamento por uma noite, um castelo por uma semana ou um condomínio por um mês: o Airbnb conecta as pessoas à experiências de viagem únicas, preços variados, em mais de 34 mil cidades e 191 países. Com um serviço de atendimento ao consumidor de nível internacional e uma comunidade de usuários em crescimento constante, o Airbnb é a maneira mais fácil de transformar seu espaço extra em dinheiro e mostrá-lo para milhões de pessoas.

O Airbnb é o maior exemplo de empresa no setor do turismo que través da tecnologia divulga os meios de hospedagens para turistas que estão em viagem à procura de alojamento, sendo uma economia compartilhada, portanto uma tendência de mercado no setor do turismo. Costa (2003) abordou sobre a tecnologia no turismo e como seriam os aplicativos para o setor do turismo: Os atuais aplicativos não foram projetados para que os prestadores de serviços turísticos on-line divulguem ou vendam produtos que não sejam os que eles mesmos fabricam. Os novos aplicativos que permitem a personalização podem realizar pesquisas automáticas por ofertas, baseadas no perfil do cliente, apresentando-as a seguir ao usuário. Além disso, entende-se que o aplicativo deve trazer a experiência, sendo alinhado com o que representa uma viagem turística (MARQUES; MOURA, 2019).

Os aplicativos, de fato, oferecem produtos de acordo com o perfil de uma gama de público, o Airbnb é um exemplo de empresa que atende a toda faixa etária e renda, oportunizando o usuário a escolher o alojamento que melhor atenda suas necessidades.

O Airbnb trabalha para facilitar o contato entre anfitrião e hospede e gerar uma melhor experiência. A Airbnb fornece uma plataforma on-line que conecta proprietários e administradores de propriedades, que têm acomodações para alugar, com os potenciais locatários que procuram alugar tais acomodações. (AIRBNB, 2018a).

O aplicativo Airbnb oportuniza e facilita o contato entre anfitrião e hospede. "Cerca de $70 \%$ de todas as mensagens dos anfitriões são enviadas pelo aplicativo, sendo que o sistema de mensagens é o recurso mais usado." (AIRBNB, 2018b, p. 1).

Portanto, o aplicativo facilita a comunicação, estando o anfitrião disponivel a qualquer momento para tirar dúvidas e gerencias as reservas, ficando assim o hospede seguro e amparado durante a viagem e podendo se comunicar a qualquer momento por mensagens pelo aplicativo. Portanto essa interação entre consumidor e anfitrião gera uma confiança e segurança para o cliente o que aumenta as chances de uma recompra ou indicação do serviço. 
O próximo capitulo abordará sobre os fatores influenciadores na intenção de recompra por meio do comercio on-line.

\subsection{INTENÇÃO DE RECOMPRA}

A intenção de recompra em uma empresa pode ser resultado de diversificados motivos, como a satisfação no serviço/produto, a qualidade, a confiança, a comodidade, entre outras (ROHDEN; MATOS; CURTH, 2016).

No que se refere a satisfação, Oliver (2010) comenta que a satisfação é o implemento do prazer, ou seja, consiste no consumo que completa as necessidades, os desejos e os objetivos do cliente, de acordo com o autor a satisfação preenche as necessidades de quem consome, sendo assim acaba gerando o desejo de recompra no serviço prestado ou no produto adquirido, tornando esse consumidor fiel a marca.

Diversos estudos analisaram diferentes fatores que influenciariam na satisfação do consumidor on-line, como a qualidade da informação disponibilizada, a qualidade do website e do serviço oferecido (MCKINNEY; YOON; ZAHEDI, 2002). A confiança na empresa também é muito importante quando falamos de recompra. A confiança do consumidor com a internet pode ser incentivo ou um obstáculo para a compra on-line (BRAMALL; SCHOEFER; MCKECHNIE, 2004).

Os autores Urban, Amyx e Lorenzon (2009) explicam sobre a confiança on-line, abordando que a transação mais intensa para a confiança é a decorrência de privacidade e segurança do site. Conforme comentado pelos autores para quem adquire um serviço ou produto nos meios on-line a confiança na privacidade do site é um fator determinante no momento da compra, pois o mesmo precisa transmitir segurança para seus clientes para gerar lealdade e fazer com que esse cliente volte a usar a empresa e indica-la para outras pessoas, mas a qualidade do canal on-line e satisfação na empresa também devem ser consideradas no momento de compra.

A confirmação de expectativas está relacionada com as etapas que os consumidores passam para às intenções de recompra, as mesmas se caracterizam da seguinte forma (BHATTACHERJEE, 2001): a) Os consumidores criam uma expectativa inicial sobre um produto ou serviço, antes de efetuar a compra; b) Após os consumidores aceitar e fazer uso de um produto ou serviço, eles formam percepções sobre o que foi consumido; c) Os consumidores avaliam o que foi consumido em relação a sua expectativa inicial e nessa etapa é confirmada sua expectativa; d) Estabelecem uma satisfação ou apreço, conforme o nível de confirmação e expectativa; e e) Clientes satisfeitos formam uma intenção de recompra, enquanto os insatisfeitos interrompem seu uso.

Portanto, o processo de confirmação de expectativas possibilita identificar as diferentes etapas que os consumidores passam e suas expectativas que influenciam na compra do produto ou serviço, demonstrando a importância da satisfação para gerar intenção de recompra. 


\subsection{UTILIDADE PERCEBIDA}

O consumidor realiza compras on-line para economizar tempo, logo a comodidade é imprescindivel, incluindo a facilidade de encontrar os produtos, o tempo gasto na compra, o prazer em comprar, a facilidade de comparar preços e os serviços pós-venda (SCHAUPP; BÉLANGER, 2005). Para Davis, Bagozzi e Warshaw (1989), a intenção do indivíduo em usar uma tecnologia é determinada principalmente por duas crenças: utilidade percebida e facilidade de uso percebida.

Em concordância com os autores, outros estudos também abordaram a importância da comodidade, praticidade e conveniência (facilidade de comprar a qualquer momento e em qualquer local com acesso à Internet), sendo os casos de Boone e Kurtz (2008) e Anckar (2003).

Com a comodidade que os apps trouxeram, pode-se realizar compras a qualquer momento pelo celular, facilitando ainda mais o acesso à Internet para realizar as compras on-line (GAO; WAECHTER; BAI, 2015). Dentre os maiores motivadores e benefícios do comércio eletrônico para os clientes, estão o acesso a grande volume de informações dos serviços/produtos acrescentando instantaneidade e flexibilidade ao processo da compra ao permitir comparações entre informações e preços dos produtos e fornecendo suporte mais consistente à decisão de compra (ANCKAR, 2003). Porém, o autor Anckar (2003) também comenta dos pontos negativos como a falta de contato pessoal nas compras realizadas pela internet. Portanto, entende-se que o e-commerce trouxe muitas facilidades como, comodidade, praticidade de comparar preços, mas ainda gera um desconforto para muitos consumidores pela falta de contato pessoal no momento da compra, porém não os impedem de utilizarem esse canal virtual.

Nesse sentido, entende-se haver uma relação positiva entre a percepção de utilidade e a intenção de recompra com o app, sugerindo a seguinte hipótese:

\section{H1: A utilidade percebida influencia na intenção de recompra de usuários em app de viagem.}

\subsection{FACILIDADE DE USO}

A compra on-line por meio de aplicativos vem aumentando, pois a facilidade de comprar sem precisar sair para uma loja física, conseguir pesquisar produtos e comparar preços é um dos maiores facilitadores para os consumidores (SCHAUPP; BÉLANGER, 2005). Segundo Davis, Bagozzi e Warshaw (1989), a facilidade de uso percebida refere-se à medida que um indivíduo acredita que ao usar uma determinada tecnologia estará livre de esforços físicos e mentais.

Todavia, a facilidade de uso no site ou aplicativo tem grande relevância no momento da compra, sendo um sistema de fácil manuseio e autoexplicativo são fatores importantes para o uso dos consumidores. Rogers (1995) comenta sobre a importância da facilidade de uso dos aplicativos para 
manuseio dos consumidores, o autor também ressalta sobre as mudanças na interface do aplicativo que pode gerar dificuldades na utilização do app. Krug (2001) também aborda sobre a importância da interface do aplicativo com um bom layout e informação para facilitar o uso dos clientes. Deste modo, a identidade visual do aplicativo deve ser clara e de fácil uso, sendo as funções autoexplicativas para a compreensão do usuário.

A facilidade de uso possui influência direta na aceitação das transações on-line (NAKAGAWA, 2008) e no comportamento de recompra de usuários em contexto on-line (VIJAYASARATHY, 2004). Dessa forma, percebe-se que a habilidade de uso das ferramentas disponíveis no contexto on-line são de grande relevância no comportamento do consumidor, dessa forma é de extrema importância um sistema de fácil manuseio e livre de esforços para facilitar o processo de compra e a diminuição de barreiras, sugerindo a seguinte hipótese:

\section{H2: A facilidade de uso influencia na intenção de recompra de usuários em app de viagem.}

\subsection{CONFIANÇA NA EMPRESA}

Os fatores tecnológicos como, segurança nas transações, usabilidade do website e privacidade dos dados pessoas são os principais geradores de confiança e satisfação do cliente com o site (SCHAUPP; BÉLANGER, 2005).

Os autores Lee e Turban (2001), citados por Perea, Monsuwe e Dellaert (2004), apontam que a falta de confiança nas compras on-line é um dos motivos que os indivíduos não comprarem no meio on-line (LEE; TURBAN, 2001; PEREA; MONSUWE; DELLAERT, 2004; ROHDEN; MATOS; CURTH, 2016). Os autores Miyazaki e Fernandez (2001) apontam que a privacidade dos indivíduos afeta na construção de relação de confiança dos consumidores no comercio eletrônico, enfatizando que quanto maior a experiência de uso na internet menor é a preocupação com a privacidade o que acaba comprometendo positivamente na decisão de compra.

Porém, devido a codificação de dados, os consumidores não consideraram a segurança na transação como um o fator mais importante (KIM; MA; KIM, 2006). Para Kim, Ma e Kim (2006) e Schaupp e Bélanger (2005), a conveniência era o aspecto mais importante para decidir comprar on-line, seguida da confiança no varejista. Avaliações, publicadas no website, de clientes que compraram na loja, a história da empresa, localização física, volume de vendas e quantidade de clientes atendidos, seriam úteis para criar confiança. Sendo assim para os clientes a opinião de outros consumidores é um dos fatores decisivos no momento da compra.

Sendo assim, sugere-se que quanto mais cresce o mercado eletrônico e quanto mais os indivíduos utilizarem esse meio para compras, maior será a utilidade percebida e facilidade de uso por eles (PALMER, 
2002; CONSTANTINIDES, 2004; ROHDEN; MATOS; CURTH, 2016) se adquire confiança no comercio eletrônico para compras, sugerindo as seguintes hipóteses:

H3a: A confiança na empresa influencia na intenção de recompra de usuários em app de viagem.

H3b: A confiança na empresa é influenciada pela utilidade percebida na utilização de app de viagem.

H3C: A confiança na empresa é influenciada pela facilidade de uso na utilização de appde viagem.

\subsection{CONFIANÇA EM TERCEIRAS PARTES}

A maior parte das empresas depende de terceiras partes que estão restritos entre a empresa e a quem consome para suprir as necessidades do consumidor. As terceiras partes são players (empresas ou pessoas) que garantem a credibilidade nas transações on-line (GARCIA; CELSO; CURTH, 2017), proporcionando maior confiança entre o fornecedor e os clientes (MCCOLE; RAMSEY; WILLIAMS, 2010; GARCIA; CELSO; CURTH, 2017). Sendo assim, é de extrema importância a confiança nos terceiros e nos dados informados na internet no momento da compra on-line. Para muitos consumidores e potenciais clientes essa falta de confiança pode evitar a finalização de determinada compra. Há muitos atores envolvidos nas compras on-line, como: bancos, certificados, empresas de cartões de crédito, e instituições de privacidade on-line do consumidor (KIM et al., 2005) e, no caso do Airbnb, hóspedes e hospedeiros. Nesse sentido, Hong e Cho (2011) apontam que a confiança dos consumidores nos intermediários tem uma grande importância nas decisões de compra no comércio virtual.

Dessa forma, entende-se haver grande importância da confiança nos intermediários no momento de decisão de compra on-line podendo ser um fator decisivo para a transação, além de ser influenciados por elementos ligados a utilidade percebida e facilidade de uso (GEFEN; KARAHANNA; STRAUB, 2003; QURESHIL; KAMAL; WOLCOTT, 2009; BUKHARI et al., 2013), pois a maior percepção de utilidade e o maior senso de controle dos usuários aumentam a confiança no intermediários reconhecidos no processo de compra, sugerindo as seguintes hipóteses:

H4a: A confiança em terceiras partes influencia na intenção de recompra de usuários em app de viagem.

H4b: A confiança em terceiras partes é influenciada pela utilidade percebida na utilização de app de viagem.

H4c: A confiança em terceiras partes é influenciada pela facilidade de uso na utilização de app de viagem. 


\section{PROCEDIMENTOS METOdOLÓGICOS}

Considerando o objetivo da pesquisa, foi realizado um estudo do tipo survey. Na aplicação da survey (por amostragem) são utilizados questionários previamente estruturados, que são compostos por questões fechadas, proporcionando o conhecimento sobre o objetivo proposto de maneira ampla (BABBIE, 1999). Além disso, a survey permite que seja utilizada uma avaliação estatística com possibilidade de cruzamento entre as variáveis que compõem o estudo (MALHOTRA, 2012).

Sendo o objetivo desta pesquisa identificar a utilidade e facilidade de uso do aplicativo Airbnb, intenção de compra e a confiança na empresa e em terceiros dos usuários do app a amostra da pesquisa é formada pelo conjunto de pessoas que utilizam ou utilizaram o aplicativo Airbnb para reserva de hospedagem. Para o levantamento dos dados, foi utilizada a técnica de amostragem não probabilística, a qual se caracteriza por ser uma técnica que o pesquisador pode selecionar a amostra arbitrariamente com base na conveniência, ou tomar uma decisão consciente sobre quais elementos incluir na amostra (MALHOTRA, 2012; HAIR JR. et al., 2009).

Com relação ao tamanho da amostra, a utilização de Modelagem de Equações Estruturais (MEE) para teste das hipóteses do trabalho sugere o incremento de respondentes relacionado ao número de parâmetros presentes no modelo (GARSON, 2012). Para Hair Jr. et al. (2009) deve-se ter de 5 a 10 respondentes por parâmetro no modelo. Dessa forma, o número de 176 respondentes válidos da pesquisa apresentou estar dentro do sugerido pela literatura.

O instrumento de pesquisa utilizou 17 questões, sendo que 15 questões verificaram os construtos da pesquisa a partir de escalas Likert de 7 pontos. Além disso, o instrumento contou com duas perguntas sobre o perfil dos respondentes (questões 16 e 17).

As questões sobre o construto Utilidade Percebida de Uso foram adaptadas de Davis, Bagozzi e Warshaw (1989), ressaltando o grau que o usuário acredita que a utilização do aplicativo irá melhorar a sua experiência de compra (UP10 - O aplicativo XX melhora minha economia na compra/reserva; UP2 - 0 aplicativo aumenta minha eficiência na compra/reserva; UP3 - O aplicativo aumenta minha habilidade de compra/reserva).

O construto Facilidade de Uso também foi adaptado de Davis, Bagozzi e Warshaw (1989), destacando o grau que o usuário acredita que a utilização do aplicativo é fácil e livre de esforços (FU1 O uso do aplicativo para compra/reserva é claro e de fácil compreensão; FU2 - O uso do aplicativo para compra/reserva não requer muitos esforços mentais; FU3 - O uso do aplicativo para compra/reserva é facilitado).

Com relação aos construtos relacionados com a confiança, o construto Confiança na Empresa foi adaptado de Mccole, Ramsey e Williams (2010), considerando o grau que o consumidor confia que 
a empresa irá cumprir as suas obrigações (CE1 - Eu sinto que minha privacidade está protegida quando realizo compras/reservas no aplicativo XX; CE2 - Eu me sinto seguro quando estou fazendo compras/ reservas no aplicativo XX; CE3 - 0 aplicativo XXX tem as características de segurança).

Da mesma forma, o construto que envolvia a Confiança em Terceiros foi adaptado dos mesmos autores, e considerou o grau que o consumidor confia nas informações disponibilizadas pelo anfitrião (CTP1 - Eu confio nas informações disponibilizadas pelos anfitriões no aplicativo XX; CTP 2 - Eu confio na imagem do anfitrião quando vou realizar a compra/reserva; CTP 3 - Eu confio que o anfitrião não utilizará minhas informações).

Por fim, o construto que abordou a Intenção de Recompra foi adaptado de Jarvenpaa, Tractinsky e Vitale (2000) e Curth (2018), destacando o grau que o indivíduo expressa a sua intenção de realizar novas transações no aplicativo (IR1 - Na próxima vez que for comprar/reservar, a probabilidade de eu escolher o aplicativo XXX é; IR2 - A probabilidade de fazer novas compras/reserva pelo aplicativo XXX é; IR3 - A probabilidade de fazer compras/reservas nos próximos anos considerando o aplicativo XX como a primeira escolha é).

A coleta de dados foi realizada considerando as seguintes etapas: a) contato inicial para apresentação da pesquisa e b) envio do link do Google Forms com as questões da pesquisa.

Para realizar a avaliação estatística dos dados coletados, foram utilizados procedimentos estatísticos de tratamentos dos dados, análise de confiabilidade e validade das questões que mediram cada construto e, por fim, a modelagem de equações estruturais (MEE) para a realização das análises estatísticas e verificação do modelo conceitual e teste de mediação de variáveis.

A operacionalização da etapa de análise de dados, contou com a utilização dos softwares Microsoft Excel ${ }^{\circledR} 2010$ e SPSS 21 (Statistical Package for Social Sciences), para análise descritiva e para preparação dos dados. Para as análises da MEE foi utilizado o AMOS ${ }^{\text {тм }} 20$ (Analysis of Moment Structures), por ser um dos principais softwares para esse tipo de análise (GARSON, 2012).

\section{ANÁLISE DOS DADOS}

Considerando a etapa descritiva, os resultados apresentaram que o perfil de respondentes apresentou que os respondentes participantes feminino possuem maior representatividade, com 52\% (102) e as faixas etárias com maior representação foram entre 20 e 29, com 59 respondentes (33,5\%) e 30 e 39 com 52 respondentes (29,5\%).

Com relação aos dados das relações dos construtos, inicialmente foi feito o tratamento dos dados e a checagem dos pressupostos. Em seguida, foram realizadas análises de confiabilidade e validade das medidas, conforme Tabela 1, com a utilização dos softwares AMOS e SPSS. 
Tabela 1 - Análise dos construtos - Alpha, CC e AVE

\begin{tabular}{|c|c|c|c|c|c|c|c|c|}
\hline \multirow[b]{2}{*}{ Construto } & \multicolumn{4}{|c|}{ Modelo de Medida Inicial } & \multicolumn{4}{|c|}{ Modelo de Medida Purificado } \\
\hline & $\begin{array}{l}\mathrm{N}^{0} \text { de } \\
\text { itens }\end{array}$ & Alpha & CC & AVE & $\begin{array}{l}\mathrm{N}^{0} \text { de } \\
\text { itens }\end{array}$ & Alpha & CC & AVE \\
\hline UP & 3 & 0,89 & 0,88 & 0,71 & - & - & - & - \\
\hline FU & 3 & 0,9 & 0,9 & 0,76 & - & - & - & - \\
\hline CE & 3 & 0,89 & 0,9 & 0,74 & - & - & - & - \\
\hline СTP & 3 & 0,89 & 0,9 & 0,75 & - & - & - & - \\
\hline IR & 3 & 0,94 & 0,83 & 0,93 & - & - & - & - \\
\hline Índices & & $>0,70$ & $>0,70$ & $>0,50$ & & $>0,70$ & $>0,70$ & $>0,50$ \\
\hline
\end{tabular}

No processo de tratamento das escalas, todos os construtos atenderam aos critérios de Alpha maior do que 0,70, confiabilidade composta maior que 0,70 e variância média extraída superior a 0,50, não havendo a necessidade de purificação (Tabela 1).

Após, foi realizado o teste da validade discriminante, comparando-se a variância média extraída (AVE) com a variância compartilhada (VC) par a par (FORNELL; LARKER, 1981). Conforme se apresenta na Tabela 2, em uma das dez combinações o valor de VC ficou maior que AVE (valores sublinhados na Tabela 2). Considerando estes casos, foi utilizado o teste de Bagozzi e Phillips (1982), que avalia a diferença de qui-quadrado para duas situações: modelo livre e modelo com correlação fixada em 1.

Tabela 2 - Análise das variâncias extraída e compartilhada

\begin{tabular}{|l|l|l|l|l|l|}
\hline \multicolumn{1}{|c|}{ Construto } & \multicolumn{1}{|c|}{ UP } & \multicolumn{1}{c|}{ FU } & \multicolumn{1}{c|}{ CE } & CTP & IR \\
\hline UP & $\mathbf{0 , 7 1}$ & & & & \\
\hline FU & $\mathbf{0 , 7 3}$ & $\mathbf{0 , 7 6}$ & & & \\
\hline CE & 0,58 & 0,6 & $\mathbf{0 , 7 4}$ & & \\
\hline CTP & 0,63 & 0,56 & 0,73 & $\mathbf{0 , 7 5}$ & \\
\hline IR & 0,08 & 0,15 & 0,12 & 0,06 & $\mathbf{0 , 8 3}$ \\
\hline
\end{tabular}

*Nota: AVEs na diagonal e a correlação nos quadrantes abaixo.

Os resultados indicaram que os construtos apresentaram diferenças de qui-quadrado superiores a 3,84 e sig. de 5\%, evidenciando validade discriminante (BAGOZZI; PHILLIPS, 1982). Após os testes anteriores, foi testado o modelo estrutural, testando-se as hipóteses propostas. Os resultados principais são apresentados na Tabela 3. 
Tabela 3 - Hipóteses do modelo conceitual

\begin{tabular}{|l|l|l|l|l|}
\hline \multicolumn{2}{|c|}{ Hipóteses } & \multicolumn{1}{c|}{$\begin{array}{c}\text { Coeficientes } \\
\text { padronizados }\end{array}$} & *Sig & \multicolumn{1}{c|}{$\begin{array}{c}\text { Suporte para } \\
\text { hipótese }\end{array}$} \\
\hline H1 & Utilidade Percebida - Intenção de Recompra & 0,26 & 0,003 & Suportada \\
\hline H2 & Facilidade de uso - Intenção de Recompra & 0,54 & 0,001 & Suportada \\
\hline H3a & Confiança na Empresa - Intenção de Recompra & 0,19 & 0,943 & Não suportada \\
\hline H3b & Utilidade Percebida - Confiança na Empresa & 0,79 & 0,001 & Suportada \\
\hline H3c & Facilidade de uso - Confiança na Empresa & 0,43 & 0,997 & Não suportada \\
\hline H4a & Confiança em Terceiros - Intenção de Recompra & 0,84 & 0,36 & Não suportada \\
\hline H4b & Utilidade Percebida - Confiança em Terceiros & 0,78 & 0,001 & Suportada \\
\hline H4c & Facilidade de uso - Confiança em Terceiros & 0,68 & 0,115 & Não suportada \\
\hline
\end{tabular}

A hipótese H1 sugeriu que a Utilidade Percebida teria efeito favorável na Intenção de Recompra. Os resultados apontam uma carga de $\beta=0,26$ e uma significância de 0,003 ( $p<0,05)$, sugerindo que quando o consumidor compreende que a utilização do aplicativo da empresa gera facilidades amplia a intenção de recompra. Para Davis, Bagozzi e Warshaw (1989) um dos fatores determinantes para usar a tecnologia é a utilidade percebida, sendo assim criando uma intenção de recompra. Conforme o autor a utilidade percebida tem grande importância para gerar fidelidade, nessa sequência, a utilidade percebida é declarada como um antecedente da intenção de recompra (LAM; HSU, 2006; LINDGREEN; WYNDSTRA, 2005; MOLINER et al., 2007; MOLINARI; ABRATT; DION, 2008).

A hipótese $\mathrm{H} 2$, que tratou a relação entre Facilidade de Uso e Intenção de Recompra foi suportada. Os resultados apontam uma boa carga na relação $(\beta=0,54)$ e uma significância de $0,001(p<0,05)$, mostrando que quando o consumidor compreende a facilidade de uso do aplicativo aumenta a possibilidade de intenção de recompra. Dessa forma, Vijayasarathy (2004) ressalta que a facilidade de uso possui uma grande influência para a recompra on-line. De acordo com Pavlou (2003), a percepção de utilidade e a percepção de facilidade de uso podem ajudar na intenção e na aceitação do comércio eletrônico, o que pode agregar na recompra do serviço. Sendo assim, a facilidade de uso de aplicativos deve ser considerada como influenciadora na recompra on-line.

A hipótese que abordou a relação entre Confiança na Empresa e Intenção de Recompra (H3a) não foi suportada, apresentando uma carga considerada baixa $(\beta=0,19)$ e significância de 0,943 , estando acima do sugerido pela literatura $(p<0,05)$. O resultado não segue os resultados encontrado por outros estudos, tanto no comportamento de compra (MCCOLE; RAMSEY; WILLIAMS, 2010), quanto no comportamento 
de recompra (ROHDEN; MATOS; CURTH, 2016), sugerindo que os fatores relevantes para a manutenção das transações no aplicativo não passam pela empresa. No entanto, percebe-se haver a possibilidade de que a confiança no provedor venha em segundo plano e que elementos que ressaltem a conveniência ou utilidade na intenção comportamental de compra (KIM; MA; KIM, 2006; SCHAUPP; BÉLANGER, 2005).

A hipótese H3b previa que a Utilidade Percebida teria efeito positivo na Confiança na Empresa. Os resultados mostraram um efeito significativo $(\beta=0,79)$ e significância dentro dos níveis sugeridos $(p<0,05)$, mostrando que quando o indivíduo entende que a utilização do aplicativo da empresa possibilita melhorias de performance na sua vida aumenta a confiança que ele terá na empresa provedora. Nesse sentido, entende-se haver uma relação direta entre os construtos relacionados à percepção de utilidade e confiança (PAVLOU, 2003; SIRDESHMUKH; SINGH; SABOL, 2002) o que também ocorre em ambientes virtuais, conforme sugere resultados deste estudo.

A hipótese H3c, referente a Facilidade de Uso e Confiança na Empresa, não foi suportada. Conforme os resultados encontrados a significância foi de 0,997 ( $p<0,05)$, mostrando que quando o indivíduo tem facilidade de usar o aplicativo não necessariamente terá confiança na empresa (SCHAUPP; BÉLANGER, 2005). O resultado segue uma linha interessante de hierarquia, sugerindo o caminho de que a facilidade não influencia na confiança que o usuário tem na empresa provedora e que a confiança deve ser influenciada por outros meios, como a utilidade, por exemplo.

A hipótese $\mathrm{H} 4 \mathrm{a}$, que previa a relação positiva entre Confiança em Terceiros e Intenção de Recompra não foi suportada, apresentando boa carga $(\beta=0,79)$, porém com significância maior do que o sugerido $0,36$ ( $p<0,05)$. Porém, estudos dos autores Hong e Cho (2011), revelaram que a confiança do consumidor em um intermediário tem uma forte influência sobre a lealdade atitudinal e as intenções de compra. No entanto, o resultado possibilita a reflexão de que o fato dos "terceiros" estarem inseridos no aplicativo para ofertarem suas hospedagens seja um preditivo de serem "confiáveis". Nesse sentido, os usuários entendem haver maior nivel de segurança na transação. Porém, isso pode ser "quebrado" quando ocorrem situações de insatisfação ou que coloquem a segurança do usuário em check.

A hipótese H4b sobre a Utilidade Percebida com efeito positivo na Confiança em Terceiros foi suportada. Os resultados demonstraram um bom efeito entre os construtos analisados $(\beta=0,78)$ e valores de significância dentro do sugerido $(p<0,05)$, demonstrando que quando o indivíduo entende que o aplicativo possibilita aperfeiçoamento nas compras se aumenta a confiança em terceiros, sendo grande importância para a decisão de compra no contexto virtual (MCCOLE; RAMSEY; WILLIAMS, 2010).

Por fim, a hipótese H4c, referente a Facilidade de Uso e Confiança em Terceiros, não foi suportada. Segundo os resultados do estudo a significância apresentada sugeriu a não existência da relação $(p<0,05)$, sugerindo que quando o indivíduo tem facilidade de usar o aplicativo não interfere diretamente 
na confiança em terceiros (VIJAYASARATHY, 2004). O resultado está alinhado com as outras relações sugeridas entre a Facilidade de Uso e os aspectos da Confiança propostos neste estudo, sugerindo que a confiança é influenciada por outros elementos e não apenas o fato de tornar um site ou aplicativo com maior facilidade de ser utilizado. Nesse sentido, entende haver a necessidade do provedor utilizar outros elementos importantes na formação de confiança, como os rankings qualificados de outros usuários, por exemplo.

Considerando os índices de ajustamento do modelo (fits), os resultados demonstraram que o estudo apresenta índices que estão dentro do sugerido pela literatura ou muito próximos. Os índices CFI $(0,87)$ e RMSEA $(0,085)$, ficaram aproximados, considerando o que é sugerido pela literatura, sendo $(>0,90)$ e $(<0,08)$, respectivamente. 0 índice TLI apresentou valor de 0,84 . No entanto, o valor encontrado não deve ser considerado um impeditivo para o modelo, pois pode ser considerado aceitável. O índice SRMR apresentou valor $(0,07)$ dentro do sugerido como aceitável pela literatura (igual ou menor do que 0,08). Por fim, o GFI apresentou valor de 0,83, o que indica o ajuste aceitável, considerando o valor sugerido de 0,90 .

\section{CONSIDERAÇÕES FINAIS}

O presente artigo pesquisou os antecedentes da intenção de recompra do aplicativo de hospedagem Airbnb, sendo a coleta de dados realizada por meio de survey com os usuários. Com a análise, foi possível identificar a influência das percepções de facilidade de uso, de utilidade percebida, confiança na empresa e intenção de recompra no aplicativo.

Os resultados demonstraram que a utilidade percebida (ou utilidade do uso), que aborda a relação da melhoria na economia por meio da compra reserva, aumenta a confiança na empresa, sugerindo que quanto mais o usuário entende que o app facilita as suas ações, maior será a confiança que ele possui na empresa provedora. O construto também influenciou positivamente na confiança em terceiras partes, sugerindo a grande importância da percepção do usuário de que o app possui importância para a performance nas ações cotidianas, possibilitando maior confianças nos provedores e nos intermediários participantes no processo de entrega, possibilitando maior possibilidade na intenção de recompra, sendo o caso do usuário pesquisado. Os resultados avançam no entendimento de que a percepção do usuário sobre a utilização possui influência positiva em diferentes elementos da confiança, o que influencia nas intenções, sendo a intenção de recompra on-line é um fator-chave de sucesso para a empresa on-line (CHOU; HSU, 2015; FANG et al., 2014). 
O resultado também avança no entendimento da relação da facilidade de uso e a confiança, sugerindo que outras variáveis possuam maior influência, sendo o caso a utilidade. Dessa forma, entende-se haver um avanço no entendimento não somente acadêmico, mas também em níveis organizacionais, sugerindo que elementos da facilidade de uso tenham a influência menor a construção da confiança e da intenção de recompra, quando comparado aos aspectos da utilidade.

Por fim, acredita-se que a pesquisa contribuiu para identificar o papel da percepção de utilidade sobre a confiança, além da influência negativa da facilidade de utilização do aplicativo para a confiança. Como limitação dessa pesquisa, a amostra foi considerada satisfatória, porém por se tratar de um assunto muito atual, no caso o uso de aplicativos mobile, acabou abrangendo um público mais jovem. Portanto, sugere-se para futuras pesquisas na área tecnológica, estudar formas de como auxiliar ou levar as facilidades tecnológicas como o uso de aplicativos para o público da terceira idade, considerando que as pessoas estão cada vez mais ligadas a tecnologia e que o público da terceira idade possui mais tempo livre e consequentemente viaja cada vez mais, seria interessante as empresas que usam aplicativos como canal de venda trazer esse público potencial para o seu comercio eletrônico. Além disso, entende-se haver a possibilidade de inclusão de inclusão de elementos que tangem a participação de outros usuários na construção do processo de confiança, sendo o caso dos usuários que avaliam a utilização anterior no próprio aplicativo ou site da empresa.

Contudo, ao analisar o comportamento de utilização das compras on-line, percebe-se que a maioria dos usuários se mostrou adepta ao comércio eletrônico e ao uso de aplicativos que requerem a disponibilização de dados pessoais. Portanto, com relação aos benefícios que a tecnologia mobile vem apresentando e promovendo uma maior facilidade na busca por produtos ou serviços de interesse e fornecendo uma melhor experiência de consumo (KOSAKA, 2009). Pode-se concluir que a tecnologia de aplicativos de hospedagem como a Airbnb traz grades benefícios e facilidades para seus usuários contribuindo no planejamento de uma viagem e na economia compartilhada.

Por fim, espera-se que este artigo possa contribuir para informações sobre economia compartilhada e a utilização de aplicativos de hospedagem, assim como as informações dos usuários do Airbnb com relação a compra por aplicativos, sendo esse um modelo atual e em crescimento no mercado tecnológico e que vem ganhando espaço e facilitando no momento de compras e planejamento de uma viagem. 


\section{Gestãoe \\ Desenvolvimento}

e-ISSN: 2446-6875

p-ISSN: 1807-5436

\section{REFERÊNCIAS}

AIRBNB. Nossa comunidade global e diversa é o que torna o Airbnb possível. 2018a. Disponível em: <https://www.airbnb.com.br/diversity>. Acesso em: 30 set. 2018.

AIRBNB. 0 novo aplicativo para celular do Airbnb ajuda você a hospedar de qualquer lugar. 2018b. Disponivel em: <https://blog.atairbnb.com/new-mobile-app-pt/>. Acesso em: 30 set. 2018.

ANCKAR, B. Drivers and Inhibitors to E-commerce Adoption: Exploring the Rationality of Consumer Behavior in the Electronic Marketplace. In: EUROPEAN CONFERENCE ON INFORMATION SYSTEMS, 11. 2003, Naples. Anais... Naples: Universidade de Nápoles Federico II, 2003. p. 41-58.

BABBIE, E. Metodologia de pesquisa survey. Belo Horizonte: UFMF, 1999.

BAGOZZI, R.; PHILLIPS, L. W. Representing and Testing Organizational Theories: A Holistic Construal. Administrative Science Quarterly, [S.I.], v. 27, n. 3, p. 459-489, 1982.

BAHL, M. Perspectiva do turismo na sociedade pós-industrial. São Paulo: Roca, 2003.

BHATTACHERJEE, A. An empirical analysis of the antecedents of electronic commerce service continuance. Decision support systems, [S.I.], v. 32, n. 2, p. 201-214, 2001.

BOONE, L. E.; KURTZ, D. L. Marketing contemporâneo. Rio de Janeiro: LTC, 2008.

BRAMALL, C.; SCHOEFER, K.; MCKECHNIE, S. The determinants and consequences of consumer trust in e-retailing: a conceptual framework. Irish Marketing Review, [S.I.], v. 17, n. 1/2, p. 13, 2004.

BRASIL. Ministério do Turismo. Celular é principal ferramenta para tomada de decisões na hora de viajar. 2018. Disponivel em: <http://www.turismo.gov.br/component/content/article.html?id=11958>. Acesso em: 30 set. 2018.

BUHALIS, D. Information and telecommunications Technologies as a strategic tool for small and medium tourism enterprises in the contemporary business environment. In SEATON, A.V. (Eds.). Tourism: The State of the Art. England: John Willey \& Sons, 1994. p. 254-274.

BUKHARI, S. M. F. et al. The antecedents of travellers' e-satisfaction and intention to buy airline tickets online: A conceptual model. Journal of enterprise information management, [S.I.], v. 26, n. 6, p. 624$641,2013$.

CHOU, S. -W.; HSU, C. -S. Understanding online repurchase intention: social exchange theory and shopping habit. Information Systems and e-Business Management, [S.I.], v. 14, n. 1, p. 19-45, 2016. 


\section{Gestãoe \\ Desenvolvimento}

e-ISSN: 2446-6875

p-ISSN: $1807-5436$

CONSTANTINIDES, E. Influencing the online consumer's behavior: the Web experience. Internet research, [S.I.], v. 14, n. 2, p. 111-126, 2004.

COSTA, R. C. E-Business para Turismo. Porto Alegre: Bookman, 2003.

CURTH, M. O. Antecedentes da Intenção de compra de participantes de comunidade Mobile. 2018. 200f. Tese (Doutorado em Administração) - Universidade do Vale do Rio dos Sinos, São Leopoldo, RS, 2018.

DAVIS, F. D. Perceived usefulness, perceived ease of use, and user acceptance of information technology. MIS Quarterly, v. 13, n. 3, p. 319-340, 1989.

DAVIS, F. D.; BAGOZZI, R. P.; WARSHAW, P. R. User acceptance of computer technology: a comparison of two theoretical models. Management science, [S.I.], v. 35, n. 8, p. 982-1003, 1989.

FANG, Y. et al. Trust, satisfaction, and online repurchase intention: The moderating role of perceived effectiveness of e-commerce institutional mechanisms. Mis Quarterly, [S.I.], v. 38, n. 2, p. 407-427, 2014.

FORNELL, C.; LARCKER, D. F. Evaluating structural equation models with unobservable variables and measurement error. Journal of marketing research, [S.I.], v. 18, n. 1, p. 39-50, 1981.

GAO, L.; WAECHTER, K. A.; BAI, X. Understanding consumers' continuance intention towards mobile purchase: A theoretical framework and empirical study-A case of China. Computers in Human Behavior, [S.I.], v. 53, p. 249-262, 2015.

GARCIA, A.; CELSO, A. M.; CURTH, M. O. A relação entre as dimensões da confiança e seus antecedentes com lealdade e boca a boca no contexto de compras on-line. In: SEMINÁRIOS DE ADMINISTRAÇÃO, 20, 2017, São Paulo. Anais... São Paulo: SEMEAD, 2017.

GARSON, G. D. Structural Equation Modelling. Asheboro, NC: Statistical Associates Publishers, 2012.

GEFEN, D.; KARAHANNA, E.; STRAUB, D. W. Trust and TAM in online shopping: an integrated model. MIS quarterly, [S.I.], v. 27, n. 1, p. 51-90, 2003.

HAIR JR., J. F. et al. Análise multivariada de dados. 6. ed. Porto Alegre: Bookman, 2009. Tradução de: Adonai Schlup Sant'Anna e Anselmo Chaves Neto.

$\mathrm{HONG}$, I. B.; $\mathrm{CHO}, \mathrm{H}$. The impact of consumer trust on attitudinal loyalty and purchase intentions in $\mathrm{B} 2 \mathrm{C}$ e-marketplaces: Intermediary trust vs. seller trust. International Journal of Information Management, [S.I.], v. 31, n. 5, p. 469-479, 2011. 


\section{Gestãoe \\ Desenvolvimento}

e-ISSN: 2446-6875

p-ISSN: 1807-5436

JARVENPAA, S. L.; TRACTINSKY, N.; VITALE, M. Consumer Trust in an Internet Store. Information Technology and Management, [S.I.], v. 1, n. 2, p. 45-71, 2000.

KIM, D. J. et al. A multidimensional trust formation model in B-to-C e-commerce: a conceptual framework and content analyses of academia/practitioner perspectives. Decision support systems, [S.I.], v. 40, n. 2, p. 143-165, 2005.

KIM, W. G.; MA, X.; KIM, D. J. Determinants of Chinese hotel customers'e-satisfaction and purchase intentions. Tourism Management, [S.I.], v. 27, n. 5, p. 890-900, 2006.

KOSAKA, V. K. I. Distribuição on-line de informações e serviços turísticos para o segmento de viajantes independentes no Brasil: 0 caso da WHL. 2009. 96 f. Trabalho de Conclusão de Curso (Monografia) - Curso de Turismo, Universidade Federal de Minas Gerais, Belo Horizonte, MG. 2009.

KRUG, S. Não me faça pensar! Uma abordagem do bom senso à navegabilidade da Web. São Paulo: Market Books, 2001.

LAM, T.; HSU, C. H. Predicting behavioral intention of choosing a travel destination. Tourism management, [S.I.], v. 27, n. 4, p. 589-599, 2006.

LEE, M. K.; TURBAN, E. A trust model for consumer internet shopping. International Journal of electronic commerce, [S.I.], v. 6, n. 1, p. 75-91, 2001.

LINDGREEN, A.; WYNSTRA, F. Value in business markets: What do we know? Where are we going? Industrial marketing management, [S.I.], v. 34, n. 7, p. 732-748, 2005.

MALHOTRA, N. K. Pesquisa de Marketing: Uma orientação aplicada. 6. ed. Porto Alegre: Bookman. 2012. Tradução de: Lene Belon Ribeiro e Monica Stefani.

MARQUES, M.; MOURA, A. F. A. O impacto da criatividade e do design na co-criação de experiências turísticas: o caso dos empreendimentos ter na região centro de Portugal. Revista Gestão e Desenvolvimento, v. 16, n. 2, p. 29-61, mai./ago. 2019.

MARTHA, G. Marketing na era digital: conceitos, plataformas e estratégias. São Paulo: Novatec, 2010.

MCCOLE, P.; RAMSEY, E.; WILLIAMS, J. Trust considerations on attitudes towards online purchasing: The moderating effect of privacy and security concerns. Journal of Business Research, [S.I.], v. 63, n. 9-10, p. 1018-1024, 2010. 


\section{Gestãoe \\ Desenvolvimento}

e-ISSN: 2446-6875

p-ISSN: 1807-5436

MCKINNEY, V.; YOON, K.; ZAHEDI, F. M. The measurement of web-customer satisfaction: An expectation and disconfirmation approach. Information systems research, [S.I.], v. 13, n. 3, p. 296-315, 2002.

MIYAZAKI, A. D.; FERNANDEZ, A. Consumer perceptions of privacy and security risks for online shopping. Journal of Consumer affairs, [S.I.], v. 35, n. 1, p. 27-44, 2001.

MOLINA, S. O Pós-Turismo. São Paulo: Alephe, 2003.

MOLINARI, L. K.; ABRATT, R.; DION, P. Satisfaction, quality and value and effects on repurchase and positive word-of-mouth behavioral intentions in a B2B services context. Journal of Services Marketing, [S.I.], v. 22, n. 5, p. 363-373, 2008.

MOLINER, M. A. et al. Relationship quality with a travel agency: The influence of the postpurchase perceived value of a tourism package. Tourism and Hospitality Research, [S.I.], v. 7, n. 3-4, p. 194-211, 2007.

NAKAGAWA, S. S. Y. A lealdade de consumidores nos ambientes de comércio online e offline. 2008. 312 f. Tese (Doutorado em Administração) - Faculdade de Economia, Administração e Contabilidade, Universidade de São Paulo, São Paulo, SP. 2008.

OLIVER, R. L. Satisfaction: a behavioral perspective on the consumer. 2.ed. New York: M.E. Sharpe, 2010.

PALMER, J. W. Web site usability, design, and performance metrics. Information systems research, [S.I.], V. 13, n. 2, p. 151-167, 2002.

PAVLOU, P. A. Consumer acceptance of electronic commerce: Integrating trust and risk with the technology acceptance model. International journal of electronic commerce, [S.I.], v. 7, n. 3, p. 101-134, 2003.

PEREA, T.; MONSUWÉ, B. G. C.; DELLAERT, K. de R. What drives consumers to shop online? A literature review. International Journal of Service Industry Management, [S.I.], v. 15, n. 1, p. 102-121, 2004.

RUIZ, T. C. D.; HORODYSKI, G. S.; CARNIATTO, I. V. A economia criativa e o turismo: uma análise do projeto soucuritiba, de Curitiba-Paraná-Brasil. Revista Gestão e Desenvolvimento, v. 16, n. 2, p. 145-169, mai./ ago. 2019.

QURESHIL, S.; KAMAL, M.; WOLCOTT, P. Information technology interventions for growth and competitiveness in micro-enterprises. International Journal of Enterprise Information Systems (IJEIS), [S.I.], v. 5, n. 2, p. 71-95, 2009.

ROGERS, E. M. Diffusion of Innovations. 4. ed. New York: Free Press, 1995. 


\section{Gestãoe \\ Desenvolvimento}

e-ISSN: 2446-6875

p-ISSN: $1807-5436$

ROHDEN, S. F.; MATOS, C. A.; CURTH, M. O. Intenções de recompra e confiança do consumidor on-line. Revista de Ciências da Administração, [S.I.], v. 18, n. 45, p. 9-24, 2016.

SCHAUPP, L. C.; BÉLANGER, F. A conjoint analysis of online consumer satisfaction1. Journal of Electronic Commerce Research, [S.I.], v. 6, n. 2, p. 95, 2005.

SIRDESHMUKH, D.; SINGH, J.; SABOL, B. Consumer trust, value, and loyalty in relational exchanges. Journal of marketing, [S.I.], v. 66, n. 1, p. 15-37, 2002.

TORRES, C. E. Palestra: Mobilidade: Computação móvel, dispositivos e aplicativos. 2011. Disponível em: <http://www.slideshare.net/cetorres/palestra-mobilidade-computao-mvel-dispositivos-eaplicativos>. Acesso em: 30 set. 2018.

TURBAN, E.; KING, D. Comércio eletrônico: Estratégia e gestão. São Paulo: Prentice Hall, 2004.

URBAN, G. L.; AMYX, C.; LORENZON, A. Online trust: state of the art, new frontiers, and research potential. Journal of interactive marketing, [S.I.], v. 23, n. 2, p. 179-190, 2009.

VASSOS, T. Marketing estratégico na internet. São Paulo: Makron Books, 1997.

VIJAYASARATHY, L. R. Predicting consumer intentions to use on-line shopping: the case for an augmented technology acceptance model. Information \& management, [S.I.], v. 41, n. 6, p. 747-762, 2004.

WU, J. J.; CHEN, Y.-H.; CHUNG, Y. S. Trust factors influencing virtual community members: A study of transaction communities. Journal of Business Research, [S.I.], v. 63, n. 9, p. 1025-1032, 2010. 